\title{
Contents, Vol. 64, 1928
}

\section{Inhalts -Verzeichnis.}

Eigenarbeiten. Seite

Avizonis, P., Über Kryptophthalmus congenitus .... 240 B¿rnbacher, Th., Über akute primäre

Irisnekrose . . . 227 Comberg, W., Prüfung der Hornhautempfindlichkeit gegen

Druckausübungam Lideals diagnostisches Hilfsmittel 127 Endres, G., siehe Wegner, W. Fischer,

F., Zur Entwicklungsgeschichte derHornhaut des

Menschen 293

Glüh, B., ExperimentelleUntersuchungen über Sehpurpur-

bildung bei Icterus am Kaninchen 69

Gredsted, A., Beitrag zur Kenntnis der Verletzungen des

Auges durch Klettenstachel 357

Gyotoku, K., Über Transplantationen von Kaninchensarkom

auf Kaninchenaugen 259

Über Metastasen bei Kaninchenaugensarkom . . 272

Hamburger, C, Bemerkung zu der Arbeit ,, Beitrag zur

Glaukosanbehandlung des Glaukoms" von Dr. Alice

Gredsted in Ztschr. f. Aug. Bd. 63, S. $85 \quad 310$

Erwiderung auf die vorstehende Bemerkung. Von

Dr. Alice Gredsted 312

Hanssen, R., Über Membranbildungen auf der Irisvorder-

fläche 278

Heller, W., Über zweiklinisch verschiedene Fälle von chro-

nischer progressiver Ophthalmoplegie $\quad 136$

Horn, L., und Rogerer, H., Über die Behandlung der tabi-

schen Optikusatrophie mit Lufteinblasung .... 377 Knüsel, 0., Vitale Färbungen am menschlichen Auge. V.

(Hierzu Tafel II-IV) 1

Rogerer, H., siehe Horn, L.

Ruhlgatz, W., Beitrag zur Extractio cataractae bei angeborener Aniridie 354

Lederer, R., Ein Versuch, den Verlauf des tabischen Seh-

nervenschwundes zu beeinflussen 50

Mandicevski, V., Über die seltenen Hornhautkomplikatio-

nen bei Conjunctivitis gonorrhoica 149

Mylius, R., Retrobulbäre Neuritis und ihre Behandlung

mit Kokain-Adrenalintamponade der Nase .... 22 Riehm, W., Die Rolle des Tuberkulins im

gesunden und

kranken Organismus 205

Roggenbau, Ch., und Wetthauer, A., Zur Frage der Erwärm- 
barkeit der einzelnen lichtbrechendenTeile des Auges

nach Bestrahlung durch einen leuchtenden Körper 143 Sa†ar, R., Cholesterinausstreuung in der vorderen Augen-

kammer als Ursache von Glaukom bei überreifem

Altersstar 46

- $\quad$ - Über basale Iridektomie bei Glaukom . . . . . 130Saltier, C. H., Scheinbares

Auswärtsschielen eines Auges

infolge abnorm großen Abstandes von Fovea undPapille 349

- $\quad$ - Haarnaht geschlitzter Tränenkanälchen .... 352

$-\mathrm{IV}-$

Selt $\beta$

Schreiber, Z., Über den abnormen Verlauf eines Netzhaut-gefäßes nebst Bemerkungen über

Kolobombildung

der Netzhaut. (Hierzu Tafel I) 65

Sgaliizer, M., Über die Art der Einwirkung der Röntgen-

strahlen bei Hirntumoren

33

Szymanski, /., Halb-Elliot. Operationstechnik ....

Wßgner, W., und Endres, G., Über die Abhängigkeit des intraokularen Druckes von der H-

Ionenkonzentration

des Blutes $\quad 43$

Weizenòlatt,Spr., Über eine eigenartige zentrale Hornhaut-

veränderung im Senium 366

Wetthaußr, A., siehe Roggenbau, Ch.

Will, H., Über einen Fall von Glaskörperblutung nach

Schädeltrauma (Fernblutung des Glaskörpers) . . 40

- - Iritis und Neuritis optici bei Weilscher Krankheit

Berichte über die deutsche ophthalmologische Literatur.

Augenoperationen (1925 und 1926). Von Dr. K. Safar in Wien . 72Untersuchungsmethoden

(I. u. II. Semester 1926). Von Doz. Dr. R.

Kramer in Wien

89

Gesellschaftsberichte.

Ophthalmolog. Gesellschaft in Wien. Sitzung vom 21. Novbr. 1927

102

Sitzung vom 28. Novbr. 1927107

Sitzung vom 12. Dezember $1927 \quad 156$

Sitzung vom 16. Januar 1928313

Sitzung vom 20. Februar $1928 \quad 388$

Berliner Augenärztliche Gesellschaft. Sitzung vom 27. Oktober $1927 \quad 112$

Sitzung vom 24. Novbr. 1927112

Sitzung vom 28. Januar 1928393

Sitzung vom 23. Februar 1928

394

12. Versammlung derDeutschen ophthalmologischen Gesellschaft in der Tschechosl. Republ., gemeinsam mit der Südostdeutschen

Augenärztevereinigung Breslau am 29. u. 30. Okt. i927inPrag 108

Sektion fur Augenheilk. der Vereinigten Med. Gesellschaft in Kiew 115

Bericht über die 28. Tag. d. Verein. mitteld. Augenärzte in Halle a. S.

II. Tag. d. Bayer, augenärztl. Ver. am 27. Novbr. 1927 in Nürnberg 177 
Ungar. Ophthalmol. Ges. in Budapest. Sitzung vom 4. Novbr. 1927197

Sitzung vom 2. Februar 1928396

Augenärztl. Gesellschaft in Hamburg. Sitzung vom 25. Novbr. 1927

31551. Versammlung des Vereins rheinisch-westfälischer Augenärzte

am 27. Novbr. 1927 in Düsseldorf

317

Bericht über die 75. Versammlung der Niederländischen Ophthalmologischen Gesellschaft.

Sitzung vom 17. und 18. Dezember 1927 32214. Sitzung der Ophthalmologischen Abteilung der Gesellschaft fürWissenschaft und Leben im Rheinisch-Westfälischen Industrie-bezirk am Sonnabend, den 5. Novbr. 1927, nachm. 4 Uhr, in der Augenklinik der Städtischen Krankenanstalten zu Essen 327

Albrecht v. Graefe zum Gedächtnis. Von C. Behr 341

Hjalmar August Schiøtz $\uparrow$. Von Harald G. A. Gjessing 344

Othmar Purtscher $\uparrow$. Von /. Meller 346

Diagnose und Therapie 117, 201,335, 403

Unfall- und Versicherungskunde 120,338

Blindenwesen 122, 340

Buchbesprechungen 124, 204, 347

Tagesnachrichten 126, 204, 407

Personalien 348

Berichtigung $\quad 348$

S. Sachregister $\quad 408$

Namenregister $\quad 414$ 